dimensions of QOL are significantly influenced by an intestinal stoma. Qualitative comments provided insight into the meanings and live experiences related to the spirituality items. Persons with ostomies report less inner peace, feeling less hopeful, not having a reason to be alive, as well as fewer spiritual and religious activities for coping. An understanding of these factors will facilitate integration of holistic care and treatment in this population.

\section{Abstract C-B1-03 \\ The Trustworthiness of Administrative Health Plan Data in Chemotherapy Research}

Erin J. Aiello Bowles, MPH, Group Health Center for Health Studies; Leah Tuzzio, MPH, Group Health Center for Health Studies; Tyler Ross, MA, Group Health Center for Health Studies; Andrew E. Williams, PhD, Kaiser Permanente Hawaii; Andrea Altschuler, PhD, Kaiser Permanente Northern California; Debra P. Ritzwoller, PhD, Kaiser Permanente Colorado; Larissa Nekhlyudov, MD, MPH, Harvard Pilgrim Health Care

Background: Collecting chemotherapy administrations is important to any study of cancer treatment. However, abstracting data from medical records can be time consuming while chemotherapy data from tumor registries may be lacking sufficient detail. The goal of this study was to describe the accuracy of automated chemotherapy data from administrative health plan databases compared with data from tumor registries. Methods: From eight Cancer Research Network (CRN) tumor registries, we identified 1218 women diagnosed with primary ovarian cancer between January 2004 and June 2006. Using the Virtual Data Warehouse (VDW), we linked cases to all known automated chemotherapy administrations through June 2006 by pooling administrative health plan data from procedure, pharmacy, and diagnosis automated databases. We then calculated the sensitivity of these three data sources for capturing any chemotherapy administrations compared to chemotherapy as coded by tumor registries at each site. Results: The health plan data contained records of at least one chemotherapy administration in $51.3 \%$ of cases (range across sites $4.3 \%-80.0 \%$ ) while the tumor registry data contained such records in $68.8 \%$ of cases (range $57.3 \%-95.2 \%$ ). The overall sensitivity of administrative data compared to tumor registry data was $69.3 \%$ (range $2.9 \%-95.2 \%$ ) and varied by administrative data source: $52.8 \%$ (range $0.0 \%-90.3 \%$ ) using procedures, $46.5 \%$ (range $0 \%-80.7 \%$ ) using pharmacy records, and 29.1\% (range $0.0 \%-65.9 \%$ ) using diagnosis codes. Sensitivity decreased over time from $81.4 \%$ among cases diagnosed January-June 2004, to $47.7 \%$ among diagnoses between January-June 2006. Conclusions: Tumor registries appeared to capture the chemotherapy administrations more often than automated health plan administrative data. We noted wide variability in the sensitivity of administrative data by site. Capture of administrative data may be enhanced through site-specific coding of chemotherapy administration and additional understanding at each site of clinical and billing coding practices.

\section{Abstract C-B1-05 \\ Development of an Instrument to Assess Comprehension of Spoken Cancer Prevention Messages}

Kathleen Mazor, EdD, Meyers Primary Care Institute; Laura Saccoccio, BS, Meyers Primary Care Institute; Douglas Roblin, PhD, Kaiser Permanente Georgia; Josephine Calvi, MPH, Kaiser Permanente Georgia; Melissa Finucane, PhD, Kaiser Permanente Hawaii; Rebecca Cowan, MA, Kaiser Permanente Hawaii; Terry Field, $\mathrm{ScD}$, Meyers Primary Care Institute; Elizabeth Dugan, PhD, Meyers Primary Care Institute; Sarah Greene, MPH, Group Health; Paul Han, MD, MA, MPH, National Cancer Institute; Mary Costanza, MD, University of Massachusetts Medical School

Background: The ability to understand orally communicated health-related messages is a critical component of health literacy. Recent attention has focused primarily on the prevalence and impact of inadequate health literacy with regard to written communication, largely ignoring that the majority of health information is communicated orally. Most Americans rely on physicians or television for health information, but there is an alarming lack of information on how well people comprehend orally presented health information. We will report on our efforts to develop and validate a computer-based assessment of health literacy-oral around cancer prevention and screening. Methods: Our methods include a multi-step test development process, beginning with iterative team reviews of the dimensions to be considered in test development, a literature review and member interviews to identify potentially confusing terms and concepts, and collection of sample messages from the media and simulated physician-patient encounters to provide a basis for the actual test items. Results: Our process identified 13 dimensions to be considered in selecting and scripting the cancer prevention and screening messages that will be included in the assessment. Four of these dimensions are (1) the purpose of the message (instructional, informational/ didactic, or query), (2) message style (narrative, statistical, or factual), (3) setting/context (messages available to the public, including those delivered via radio, television, or internet; messages delivered to the individual including messages delivered in physician-patient encounters, or in person or telephone encounters with other healthcare providers), and (4) content (e.g., cancer causes, screening, risk, epidemiology). The presentation will include full descriptions of all 13 dimensions with illustrative examples, the results of the iterative test specification process and sample items. Conclusions: We have successfully completed the first steps in developing a test to assess comprehension of health literacy oral in the context of cancer prevention and screening. The next steps include soliciting feedback on our methods and results to date, and suggestions for pilot testing and refining our materials.

\section{Analytic Methods}

\section{Abstract C-B3-01 \\ Data Quality Assessment of the Vaccine Safety Datalink Dynamic Data Files}

Allison L. Naleway, PhD, Kaiser Permanente Center for Health Research; Lois Drew, Kaiser Permanente Center for Health Research; Brad Crane, MS, Kaiser Permanente Center for Health Research; James Baggs, PhD, Centers for Disease Control and Prevention; Eric Weintraub, Centers for Disease Control and Prevention; John P. Mullooly, PhD, Kaiser Permanente Center for Health Research

To enhance our ability to rapidly detect adverse events following immunization, the Vaccine Safety Datalink (VSD) developed dynamic data files (DDF). The DDF are created through weekly extracts of selected electronic medical records data at participating VSD HMOs. However, the timeliness and completeness of these files depend largely on the data storage capacity and infrastructure at each of the HMOs. VSD Data Managers produce a series of weekly, monthly, and annual reports to identify patterns that might signal a data quality problem in the DDF. All sites collect multiple weeks of event data during the weekly data extracts. To assess data quality, we can examine summaries of any particular week of event data repeated over multiple extracts. One data quality report summarizes the number of observations in a dataset for a given event week across multiple extracts, and calculates the percent change in observations across extracts. We used this report to estimate the number of weeks (or 'lag time') until the diagnosis and vaccination data at each HMO become relatively stable in the DDF. For each event week, we estimated the lag time until stability for outpatient diagnoses, inpatient diagnoses, and vaccinations in the DDF. Stability was defined as four consecutive extracts with less than $1 \%$ change (positive or negative) from the previous week. We created a set of criteria to define anomalous extract weeks and then imputed the percent change for these anomalous weeks in our estimation of lag time. Anomalous weeks included extracts in which datasets were not updated (i.e., the percent change across event weeks was $0 \%$ ) and extracts in which the percent change across all event weeks was equal to $5 \%$. The average lag time for outpatient diagnoses ranged from 3 to 19 weeks, and the average lag time for inpatient diagnoses ranged from 3 to 25 weeks across HMOs. The average lag time for vaccinations ranged from 2 to 26 weeks. Our findings suggest that the timeliness of diagnosis and vaccination data varies across the HMOs. In spite of this limitation, the DDF have proven to be a useful tool for rapid vaccine adverse event detection. Continuous data quality assessment of the DDF enables problems to be identified rapidly and either corrected or properly documented. 